\title{
Management des Protheseninfekts
}

\author{
Christian Friesecke, Joachim Wodtke
}

\section{Zusammenfassung}

Die periprothetische Infektion stellt ein dauerhaftes Erkrankungsrisiko in der Endoprothetik dar. Die zielgerichtete und adäquate Diagnostik unter optimalen Voraussetzungen ist eine Bedingung für die erfolgreiche Therapie dieser schweren Komplikation. Anamnese, Klinik und eine mögliche CRP-Erhöhung ergeben die Verdachtsdiagnose. Eine unter aseptischen Kautelen durchgeführte Gelenkpunktion und die Untersuchung der Probe in einem geeigneten mikrobiologischen Labor beweisen durch Keimnachweis die Diagnose. Die Resistenzbestimmung liefert die Voraussetzung für eine gezielte Behandlung. Diese besteht in der Regel im Austausch der Prothese sowie einem radikalen Débridement in Verbindung mit einer topischen und systemischen Antibiotikatherapie. Der Prothesenaustausch kann ein- oder zweizeitig erfolgen. Das einzeitige Vorgehen bietet entscheidende Vorteile für Arzt, Patient und Kostenträger, bei gleicher Chance auf Infektsanierung und besserem funktionellen Ergebnis.

\section{Management of Periprosthetic Infection}

The periprosthetic infection is a constant risk and a severe complication in joint arthroplasty. Systematic diagnostics under optimal conditions are necessary to establish a successful therapy. The patient's history, clinical examination and an elevated CRP-level provide the basis for the suspicion of an infection. The diagnosis is confirmed by identification of the pathogen from a sample collected through joint aspiration under sterile conditions. The microbiological examination is carried out in a laboratory that is specialised in the diagnosis of foreign body infections. The pattern of resistance of the identified pathogen determines the topical and systemic course of antibiotics to be used. The surgical treatment is characterised by exchange of the prosthesis and a radical debridement. The exchange can be done in one or two stages. The onestage exchange offers several advantages in comparison to two or more stage procedures for all those involved - patient, surgeon and the health-care system - while providing the same results in successful elimination of the infection, with an even better functional outcome.

\section{Definition und Einleitung}

Die periprothetische Infektion stellt ein eigenes Krankheitsbild dar, das mit der Implantation von Fremdkörpern entstand. Sie ist definiert durch die Besiedlung der Implantatoberfläche mit Keimen und bedarf einer speziellen Diagnostik und Therapie.

OP-JOURNAL 2010; 26: 38-45

(c) Georg Thieme Verlag KG Stuttgart · New York DOI http://dx.doi.org/10.1055/s-0030-1250001

Ursächlich ist ein Keimeintrag während der Operation oder später eine hämatogene bzw. lymphogene Streuung. Ein zuverlässiges klinisches Leitsymptom gibt es nicht. Die Erkrankung kann völlig asymptomatisch verlaufen, gelegentliche Schmerzen verursachen, aber auch Entzündungszeichen im Narbenbereich zeigen, bis hin zu Fistelbildung oder großflächigen Weichteildefekten. In seltenen Fällen kann es zu hochakuten, lebensbedrohenden, septischen Verläufen kommen, auch mit letalem Ausgang.

\section{Theoretische Grundlagen}

In Anwesenheit von Fremdkörpern gehen Bakterien von der planktonischen Form in die sessile über und besiedeln die Implantatoberfläche. Dabei bilden sie zusätzlich einen Biofilm [6,18], der die Wirksamkeit sowohl der körpereigenen Abwehr als auch von Antibiotika negativ beeinflusst.

Diese Tatsache ist die Erklärungsgrundlage dafür, dass schon eine sehr geringe Anzahl von Bakterien in der Lage ist, die Infektion auszulösen und zu unterhalten $[14,17,37,38]$.

Der Grund für das Persistieren einer Infektion bei ausschließlich antibiotischer bzw. periprothetischer chirurgischer Intervention wird darin gesehen, dass die Biofilm-Schutzzone nicht erreichbar ist.

Eradikation ist nur möglich durch kompletten Austausch der besiedelten Fremdmaterialien.

Die Kontamination der Prothesenoberfläche vollzieht sich entweder während des operativen Eingriffs oder später durch hämatogene oder lymphogene Verbreitung aus Infektionsherden im Körper. Besonders nach gastrointestinalen Operationen, Enteritiden, größeren Zahnbehandlungen [23], eitrigen Entzündungen wie z.B. Panaritien, Sinusitiden und insbesondere Erysipelen nach Bagatellverletzungen kann eine Spätinfektion durch Streuung die Folge sein. Eine Diszitis kann, entsprechend dem tuberkulösen Senkungsabszess, die Infektion des prothetisch versorgten Hüftgelenks per continuitatem verursachen.

\section{Diagnostik}

\section{Anamnese}

Alle im Zusammenhang mit der vorangegangenen prothetischen Versorgung aufgetretenen Wundheilungsstörungen 


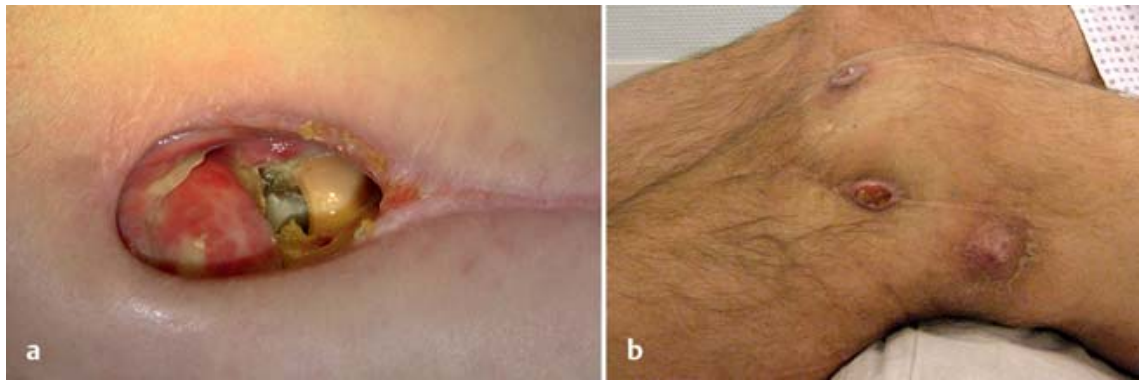

Abb. $1 \mathbf{a}$ und $\mathbf{b}$ a Weichteildefekt über dem Hüftgelenk mit freiliegender Prothese. b Fistel über dem Kniegelenk mit ungünstiger Lokalisation.

oder Revisionseingriffe sind hochverdächtig auf das Vorliegen einer periprothetischen Infektion. Oft berichten diese Patienten, niemals beschwerdefrei geworden zu sein. Aber auch Patienten, die jahrelang beschwerdefrei mit ihrer Prothese waren und nun über plötzlich neu einsetzende Schmerzen klagen, sind infektverdächtig. Dabei ist gezielt nach vorangegangenen Operationen, Zahnbehandlungen, entzündlichen Erkrankungen, Fieberschüben und Nachtschweiß zu fragen.

Jedes mit auch nur leichten Beschwerden im prothetisch versorgten Gelenk verbundene Fieber ist abklärungsbedürftig durch Gelenkpunktion vor einer antibiotischen Behandlung im Sinne einer Ausschlussdiagnostik. Die ungezielte Antibiotikagabe kann das Untersuchungsergebnis des Punktats - sehr zum Schaden des Patienten - erschweren oder unmöglich machen und muss als Behandlungsfehler bezeichnet werden.

\section{Klinik}

Die klassischen Symptome Rubor, Dolor und Calor sind wegweisend. Eine Fistel mit Gelenkkontakt sowie Weichteildefekte mit freiliegenden Prothesenteilen bedeuten immer eine periprothetische Infektion (Abb. 1). Nur der Keimnachweis muss noch erfolgen. Nicht selten - ca. $30 \%$ an der Hüfte und ca. $10 \%$ am Knie [12] - finden sich aber völlig unauffällige Weichteilverhältnisse.

Im Einzelfall muss die Differenzierung zwischen subkutanem Weichteilinfekt und periprothetischer Infektion gelingen. Dabei sind reine Subkutaninfekte in der direkten postoperativen Phase häufiger als im Langzeitverlauf nach prothetischer Versorgung. Hier bedeuten sie eher einen Hinweis auf eine tiefe Infektion.

\section{Keimnachweis und Resistenzbestimmung}

Das entscheidende diagnostische Mittel in der Diagnose der periprothetischen Infektion ist die Gelenkpunktion.

Sind Fisteln oder größere Weichteildefekte vorhanden, wird sie ergänzt durch ein Abradat. Abstriche sind nicht geeignet, da die Keime in der Fistelwand zu finden sind. Der Keimnachweis aus diesem mit Gelenkkontakt gewonnenen Untersuchungsmaterial ist der Beweis für das Vorliegen einer periprothetischen Infektion. Eine umfassende Testung des isolierten Keimes liefert das Antibiogramm, welches die Grundlage der topischen und systemischen Antibiotikatherapie darstellt.
Die Punktion erfolgt unter streng aseptischen Kautelen im Operationssaal und, wenigstens beim Hüftgelenk, unter Bildwandlerkontrolle [12] (Abb. 2). Diese erleichtert es dem Operateur, ein sicheres Punktat aus dem Gelenkbinnenraum zu gewinnen und erspart dem Patienten unnötige Schmerzen bei der Suche mit der Kanülenspitze nach dem Prothesenkontakt.

Eine Lokalanästhesie darf nicht verwendet werden, weil die Wirkstoffe bakterizid sind. Eine Verdünnung mit Kochsalz ist ebenso kontraindiziert.

Bei optimalen Bedingungen in einem auf die Diagnostik von Fremdkörperinfektionen spezialisierten Labor liegt die Spezifität der Punktion bei 90\% [16,31, 32]. Es soll mindestens $1 \mathrm{ml}$ Punktat gewonnen werden. Der Transport ins Labor muss schnell und unter anaeroben Bedingungen erfolgen. Die Proben müssen mindestens über eine Zeit von 2 Wochen im Labor bearbeitet werden. Bei fehlendem Keimnachweis in der Kultur und mikroskopischem Befund mit Nachweis von Leukozyten mit Phagozytose besteht ein hoher Infektverdacht und die Punktion ist $\mathrm{zu}$ wiederholen. Sollten bereits Antibiotikagaben erfolgt sein, ist eine Karenz von 4 Wochen vor der Punktion anzustreben. In Einzelfällen kann auch eine Probeexzision indiziert sein. Ist die Infektion durch den Keimnachweis bewiesen, sind weitere kostenträchtige Untersuchungen nicht erforderlich.

\section{Labor}

Der Leitparameter der Labordiagnostik ist das CRP.

Es ist in ca. 84\% der Fälle erhöht, wenn auch häufig nur leicht. Eine fehlende CRP-Erhöhung schließt eine Infektion also keineswegs aus. Die heute nur noch
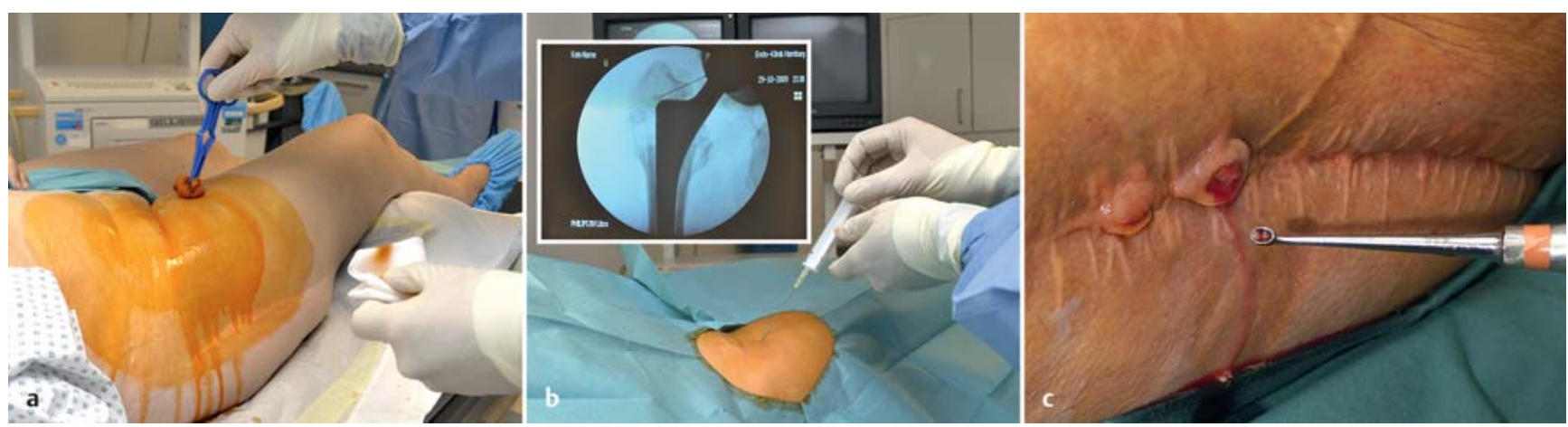

Abb. 2 a bis c a Großflächige Desinfektion, b Punktion unter Bildwandlerkontrolle im Punktionsraum und c Abradatgewinnung mit dem scharfen Löffel. 
selten bestimmte BSG ist ebenfalls in $80 \%$ erhöht, während sich erhöhte Leukozytenzahlen nur in 28\% der Fälle [35] finden.

\section{Bildgebung}

Ein Nativröntgenbild ist für die Operationsplanung unerlässlich. Hinweise auf eine Infektion zeigt es nur in wenigen Einzelfällen in Form von typischen infektbedingten heterotopen Ossifikationen, die sich auch häufiger nach mehrfachen frustranen Weichteilrevisionen einstellen (Abb.3). Die verschiedenen Szintigrafien helfen nicht weiter. Sie sind in ihrer Spezifität zu schlecht $[21,28]$. Zusätzliche klinisch stumme Foci sind besser mittels PET-CT zu finden. Diese kann präzise die infizierten Strukturen darstellen. Damit trägt sie zwar nicht zur Diagnose bei, kann aber für den Operateur wertvolle Hinweise auf die Ausdehnung der Infektion und die sich daraus ergebende Operationstaktik geben (Abb. 4).

\section{Therapie}

Die therapeutischen Konzepte in der Behandlung der periprothetischen Infektion ergeben sich aus ihren theoretischen Grundlagen:

\section{Prothesenerhalt bei gesichertem epifaszialen Infekt}

Bei einem subkutanen Abszess, ausgehend von einer Fadenfistel oder einer Stichkanalinfektion, kann der Prothesenerhalt gelingen, wenn sich bei der Revision eine eindeutig intakte Faszienbarriere findet. In dieser Situation droht akut ein Übergreifen auf das Implantat, sodass schnell und sicher diagnostiziert und die Behandlung erfolgen muss. Die Revision ist dann zu begrenzen auf den epifaszialen Raum und kann z.B. mittels Sekundärnaht oder Vakuumversiegelung erfolgen.

Selbstverständlich muss präoperativ das Gelenk punkiert werden, um sicherzustellen, dass keine periprothetische Infektion vorliegt. Dabei darf keinesfalls durch das infizierte Areal hindurch punktiert werden.

Prothesenerhalt durch Saug-Spül-Drainage und Débridement beim Akutinfekt

Tritt bei Primäreingriffen im Verlauf von 2 bis 3 Wochen ein Akutinfekt auf, kann eine Revision mit Infektsanierung unter
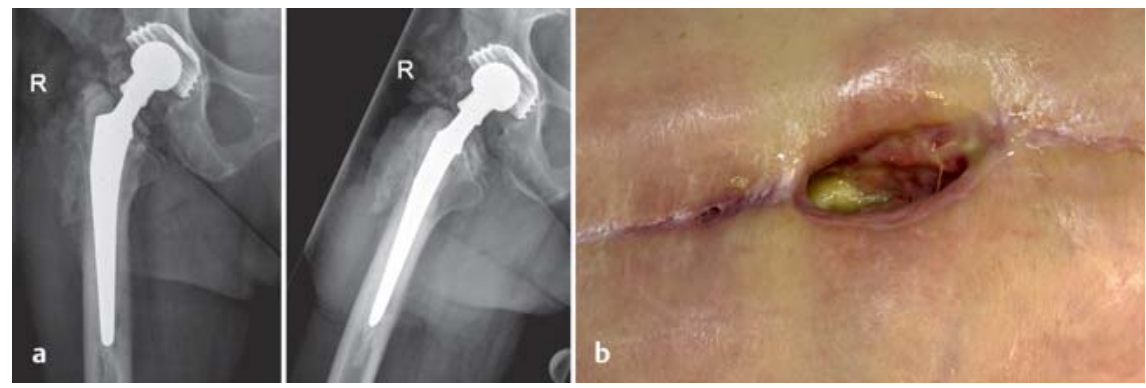

Abb. $\mathbf{3} \mathbf{a}$ und $\mathbf{b}$ a Infektinduzierte Ossifikationen nach Mehrfacheingriffen und b Weichteilsituation beim selben Patienten.

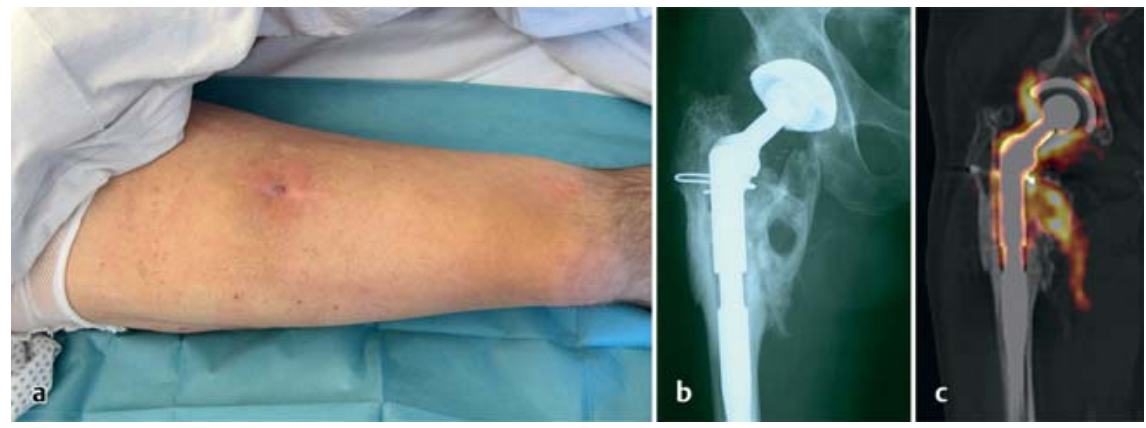

Abb.4a bis c a Ventrale Oberschenkelfistel, b Röntgenbild und c PET-Darstellung mit Nachweis einer Infektstraße zur Ossifikation.

Prothesenerhalt gelingen. Die Erfolgsrate liegt bei $50-60 \%$, wenn der Eingriff von einem radikalen Débridement, Jet-Lavage des Operationsgebiets, einer SaugSpül-Drainage über 3-4 Tage, aber nicht länger, und einer systemischen Antibiotikatherapie entsprechend des spezifischen Antibiogramms begleitet wird $[8,30]$.

Entscheidend für den Erfolg der Methode sind der frühestmögliche Zeitpunkt der Revision, die Radikalität des Débridements und die Kenntnis der Keimlage.

Dass die Verankerungsstrecke, also die knöcherne Substanz, dabei nicht beeinträchtigt wird, ist ein entscheidender Vorteil dieser Methode. Mehr als 3 Wochen nach dem Primäreingriff fällt die Erfolgsrate dieser Therapieform steil ab $[7,30]$, was sich aus der jetzt abgeschlossenen Besiedlung der Prothesenoberfläche erklären lässt [6].

\section{Prothesenwechsel}

In allen anderen Fällen von periprothetischen Infektionen ist der Austausch des Implantats samt Débridement der infizierten Weichteile und des knöchernen Prothesenlagers notwendig, um eine Infektsanierung erreichen zu können.
Dabei ist es unerheblich, ob die Prothesenteile gelockert oder noch fest verankert sind $[4,22,35,37]$. Der Eingriff wird ergänzt durch eine spezifische topische und systemische Antibiotikatherapie. Einzeitiges und mehrzeitiges Vorgehen basieren dabei auf dem gleichen Prinzip, nämlich der Entfernung des infizierten Fremdkörpers. Das einzeitige Vorgehen ist als Goldstandard anzusehen, da es gegenüber dem zweizeitigen Vorgehen Vorteile für den Patienten, den Chirurgen und die Kostenträger bietet. Voraussetzung für den Erfolg des einzeitigen Vorgehens sind allerdings die präoperative Keimbestimmung und ein Resistenzmuster, das eine topische und systemische Antibiotikatherapie erlaubt, ein erfahrenes Operationsteam und die enge Zusammenarbeit mit einem klinisch tätigen, spezialisierten Mikrobiologen.

\section{Einzeitiger Wechsel}

Die Vorteile des einzeitigen Vorgehens ergeben sich aus der Vermeidung sowohl eines 2. operativen Eingriffs gleicher Größenordnung als auch des prothesenlosen Intervalls. Die Psyche des Patienten und die Weichteile im Operationssitus werden nur einmal belastet. Alle operativen Komplikationen wie Thrombose, Embolie, Gefäß- oder Nervenschäden, Wundheilungsstörungen, neuerliche Infektio- 
nen oder die Gefahren der Bluttransfusion können nur einmal auftreten und sind also um 50\% vermindert. Für den Chirurgen ist es operationstechnisch erheblich einfacher, nach erfolgter Prothesenentfernung und Débridement sofort zu reimplantieren, als Wochen oder Monate später in einem durch Narbenbildung veränderten Situs.

Die Weichgewebe werden geschont, weil das erforderliche 2. Débridement anlässlich der Reimplantation vermieden wird. Daraus resultiert ein besseres funktionelles Ergebnis [22].

Und schließlich bietet das einzeitige Vorgehen auch Vorteile für die Kostenträger. Da hinsichtlich des Erfolgs der Infektsanierung zwischen beiden Vorgehensweisen keine signifikanten Unterschiede bestehen, ist das einzeitige Vorgehen sowohl unter medizinischen als auch ökonomischen Gesichtspunkten als überlegen anzusehen $[1,8,9,19,20,22,27,28$, 36].

Die topische Antibiose erreicht sehr hohe lokale Wirkstoffspiegel, die bei systemischer Verabreichung ohne toxische Nebenwirkungen nicht erreicht werden können.

Dazu werden dem Knochenzement jeweils entsprechend dem individuellen Antibiogramm geeignete Antibiotika in Pulverform beigemengt. Dabei kann die Pulvermenge 10\% der Zementmenge erreichen, ohne relevante Veränderungen der mechanischen Zementeigenschaften zu verursachen [24,33] (Abb. 5).

Es gibt auch die Möglichkeit, mit Vancomycin imprägnierten Fremdknochen zur topischen Antibiose mit einer zementfreien Prothese zu kombinieren, wobei die Zahlen in der Literatur klein sind und das Verfahren kaum verbreitet ist $[5,34]$.

Die topische Antibiose ist allein nicht geeignet, die Infektion zu sanieren. Dieses wird durch das radikale chirurgische Débridement angestrebt. Jedoch ist immer davon auszugehen, dass noch eine Restkontamination im Operationsgebiet vorliegt, obwohl eine Abreicherung der Keimlast um mehrere Zehnerpotenzen erwartet werden kann. Diese verbliebenen Keime sollen durch die topische Antibiose eliminiert werden, wobei v.a. eine erneute Besiedlung der Prothesenoberflächen verhindert und der Ausbildung eines Biofilms vorgebeugt werden soll.

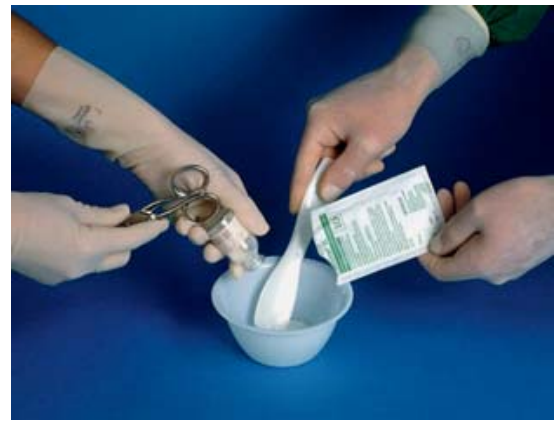

Abb. 5 Manuelle Zementzubereitung zur Herstellung der spezifischen, individuellen, topischen Antibiotikamischung.

Deshalb ist nach unserer Auffassung die zementierte Verankerung der neuen Prothese erforderlich, da man andernfalls auf das bakterizide Potenzial der topischen Antibiose komplett verzichtete.

Buchholz und Engelbrecht [2] begannen 1969, als die Infektionsraten der primären Hüftendoprothetik noch im Bereich von $10 \%$ lagen, dem Knochenzement Antibiotika beizumengen. Dadurch gelang es, die Infektraten deutlich zu senken [8]. Es entstand der Refobacin-Palacos. Umfangreiche Studien über die Thermostabilität verschiedener Antibiotika und ihre Elutionseigenschaften aus verschiedenen Zementsorten ermöglichten eine Auswahl von geeigneten Antibiotika [25,33]. Auf dem Boden dieser Erkenntnisse entwickelte Buchholz kontinuierlich das Konzept des einzeitigen Wechsels bei periprothetischer Infektion, wobei unter antibiotischem Schutz sofort reimplantiert wurde. Es ist bereits 1972 [3,4] veröffentlicht worden und wird seither in kaum veränderter Weise erfolgreich angewandt.

Die systemische Antibiotikatherapie ist nur als adjuvant anzusehen und nur dort wirksam, wo eine gute Durchblutung besteht.

Sie wird in der Regel über etwa 2 Wochen postoperativ durchgeführt. Die Entscheidung über die Dauer richtet sich im Einzelfall in Absprache mit dem klinischen Bakteriologen nach der Keimlage, den Wundverhältnissen und dem Verlauf der CRP-Werte.

\section{Zweizeitiger Wechsel}

Ein zwei- oder mehrzeitiges Vorgehen mit Entfernung der Implantate und Débridement im 1. Schritt, einer antibiotischen Interimstherapie durch antibiotikahaltige Spacer sowie systemische bzw. orale Antibiotikagabe über Wochen oder Monate, gefolgt von einem 2. Débridement und der Reimplantation, kann in Einzelfällen indiziert sein.

Bei unbekannter Keimlage wäre eine gezielte topische Therapie nicht möglich, bzw. nur zufällig wirksam. Bei Resistenzen der Erreger oder Allergien der Patienten gegen die erforderlichen Antibiotika ist ebenfalls ein zweizeitiges Vorgehen sinnvoll, wobei das chirurgische Débridement hier allein im Vordergrund steht. Ebenso sollte ein zweizeitiges Vorgehen erwogen werden, wenn das chirurgische Débridement nicht sicher radikal genug durchgeführt werden kann, wie z. B. beim offenen Beckenboden im Hüftgelenksbereich. Hier können die dort liegenden anatomischen Strukturen ein ausreichend radikales Débridement kompromittieren.

Der Vorteil des zweizeitigen Vorgehens in diesen Fällen ist jedoch nicht in einer höheren Chance auf Infektsanierung zu sehen, sondern nur in der Erleichterung des Folgeeingriffs im Falle einer Infektpersistenz, weil keine Prothesen- und Zemententfernung nötig ist und evtl. jetzt die Keimlage geklärt ist.

Wiederholte Weichteilrevisionen mit oder ohne Spülung und Einlage von Antibiotikaträgern, mit oder ohne Wechsel von Polyäthylenteilen, aber ohne Prothesenentfernung, müssen unbedingt vermieden werden.

Sie bieten kaum Chancen auf Infektsanierung und schädigen das Weichgewebe erheblich. Daraus ergeben sich zwangsläufig schlechte funktionelle Resultate mit erheblichen Belastungen für den Patienten und hohen Kosten für die Allgemeinheit.

Wodtke [35] fand bei 1000 Patienten mit periprothetischer Infektion, dass mehr als $60 \%$ schon 1 - bis 4 -mal wegen der Infektion operiert worden waren vor der schließlich sanierenden Therapie, und bei der Hälfte der Patienten die Infektion bereits seit länger als einem halben Jahr bestanden hatte. Dieses sind Indizes für eine suboptimale Betreuung dieser besonders betroffenen Patienten.

\section{Resektion}

Sie ergibt sich heute in erster Linie aus irreparablen Substanzverlusten durch die vorangegangenen Operationen. Eine nochmalige das funktionelle Ergebnis 
und die Verankerung einer Prothese betreffende sinnvolle Rekonstruktion mit einer Endoprothese ist dann gelegentlich, besonders im Azetabulum, nicht mehr möglich. Die Resektion stellt aber keine eigenständige Therapiealternative zur Infektbeherrschung dar.

\section{Ablation}

Bei nicht beherrschbaren Infektzuständen und endgradig geschädigtem Weichteilmantel bleibt in Einzelfällen nur die Exartikulation im Hüftgelenk bzw. die Oberschenkelamputation, falls das Kniegelenk betroffen ist. Zur Wiederherstellung einer lebenswerten Situation ohne Infekt, Fisteln und Schmerzen kann sie erfolgreich sein.

\section{Suppression}

Stets ist sorgfältig abzugrenzen, ob ein schlechter Allgemeinzustand durch $\mathrm{Ne}$ benerkrankungen oder durch das Infektgeschehen verursacht wird. Bei drohender oder bestehender Sepsis ist ohnehin die operative Intervention, und sei es nur im Sinne einer Entlastung des Infektionsherds, durch Eröffnung unumgänglich, da allein kausal therapierend und ggf. lebensrettend. Gelegentlich verbietet aber tatsächlich die Multimorbidität den Eingriff, da eine Gefahr quo ad vitam durch die Operation befürchtet werden muss, die weit über dem zu erzielenden Nutzen liegt.

In diesen Fällen ist eine Langzeitantibiose zur Unterdrückung der Symptome der periprothetischen Infektion möglich. Dies gilt auch für Patienten, die eine operative Therapie hartnackig ablehnen [15]. Sie kann aber nicht zur Infektsanierung führen.

Die Gefahr der Langzeitsuppression besteht in besonders ausgedehnten Schädigungen an den Weichgeweben und der Knochensubstanz durch die sich schleichend ausbreitende Infektion.

Teilweise ist später doch eine Revision zwingend erforderlich unter wesentlich schlechteren Ausgangsbedingungen. Zudem können sich Antibiotikaresistenzen entwickeln. Die Entscheidung für eine Langzeitantibiose muss daher sehr sorgfältig abgewogen werden.

\section{Operationstechnik}

Die Operation lässt sich in eine 1. „septische" Phase und die daran anschließende 2. „saubere“ Phase gliedern.

Die 1. Phase betrifft das Débridement und die restlose Entfernung aller Fremdmaterialien, also der Prothese, des Zements, etwaiger Osteosynthesematerialien wie Schrauben, Platten, Drähten etc., aber auch alter, nicht resorbierbarer Nahtmaterialien.

Das Débridement soll standardisiert und systematisch erfolgen, um sicherzustellen, dass alle Areale des Situs wirklich erreicht werden.

Ohne Standardisierung ist die Gefahr groß, infizierte Areale zu belassen, da nach längerem Verlauf des Eingriffs eine makroskopische Unterscheidung zwischen debridierten und nicht debridierten Arealen schwierig sein kann. Das Débridement soll nicht mit einer Kürette erfolgen, sondern es müssen mit der Schere oder dem Skalpell die gesamten Infektmembranen, die eine erhebliche Schichtdicke erreichen können, möglichst zusammenhängend entfernt werden.

Am Hüftgelenk empfiehlt sich, bei intaktem Weichteilmantel, der dorsale $\mathrm{Zu}-$ gang, da er den besten Überblick ermöglicht und, je nach Erfordernis, sowohl nach distal als auch nach proximal unbegrenzt erweiterbar ist. Im Kniebereich weist der Subvastuszugang den unschätzbaren Vorteil auf, den gesamten Streckapparat unangetastet zu lassen.

Liegt eine Fistel oder eine Wunddehiszenz vor, ist selbstverständlich der $\mathrm{Zu}$ gang in ihrem Verlauf zu wählen, um den Fistelkanal exzidieren zu können. Häufig betrifft dies am Hüftgelenk ventrale Gelenkzugänge. Dann findet sich, besonders wenn zusätzliche Weichteileingriffe durchgeführt wurden, eine Schädigung bis hin zum kompletten Verlust der Glutaeus-medius-Muskulatur am Trochanter major. Daraus resultiert ein dauerhaft positives Trendelenburg'sches Hinken sowie eine dauerhafte Luxationsneigung, da der Schaden irreparabel ist. Ähnlich ist nach anteriorem Zugang zum Kniegelenk gelegentlich die Strecksehne durch die Infektion oder wiederholte Revisionen zerstört. Dann ist der Erhalt einer stabilen Streckfunktion nicht mehr zu erreichen und eine Arthrodese erforderlich.
Während der gesamten Operation soll die sekundäre Kontamination, also die iatrogene Verschleppung von Infektmaterial, so weit wie möglich begrenzt werden.

Deshalb wird nach Ablösen der Außenrotatoren das Operationsgebiet mit feuchten Bauchtüchern geschützt und erst dann die Eröffung des Gelenkbinnenraums vorgenommen. Das Débridement beginnt unter sorgfältiger Schonung des $\mathrm{N}$. ischiadicus dorsal bis auf den Pfannenrand. Dann wird nach kaudal bis auf die Psoassehne und nach proximal bis auf den Pfannenrand sämtliches Infektmaterial entfernt. Nach Luxation des Gelenks und Entfernung der Schaftprothese wird das Weichteildébridement nach ventral komplettiert. Dabei müssen im Verlauf der Psoassehne am vorderen Pfannenrand in das kleine Becken ziehende Infektstraßen unter Schonung der Gefäße entfernt werden.

Die Radikalität des Débridements ist entscheidend für eine erfolgreiche Infektsanierung.

Dabei sollen infizierte Strukturen, auch knöcherne, also z.B. Sequester, komplett reseziert werden. Die Entscheidung zwischen erforderlicher Radikalität in der Resektion und Erhalt von Strukturen erfordert ein hohes Maß an Erfahrung beim Operateur. Er muss ein Gespür entwickeln für versteckte Infektnester. Im Bereich von knöchernen Perforationen im Femur muss eine Revision mit Ablösung der umgebenden Vastusmuskulatur durchgeführt werden, um hier häufig lokalisierte Abszesse nicht zu übersehen. Andernfalls ist die Infektpersistenz vorprogrammiert.

Insgesamt gilt: Im Vordergrund steht die Infektsanierung.

Die Entfernung der Implantate kann teilweise extrem anspruchsvoll sein. Die Nomenklatur der septischen Prothesenlockerung ist dabei irreführend, denn meist sind die Implantate noch fest im Knochen verankert [36]. Oft ist die Entfernung der fest verankerten Prothesenteile nur unter zusätzlichen Knochensubstanzverlusten möglich. Dieses gilt besonders für zementfreie Implantate mit grob strukturierten Oberflächen oder Sternprofil.

Selbstverständlich muss im Hinblick auf Funktion und Prothesenstandzeit der intraoperative Knochensubstanzverlust so gering wie möglich gehalten werden. 
Eine zementierte Schaftprothese lässt sich aus dem Zementköcher ausschlagen. Die Zementreste sind mit speziellen Meißeln und Extraktoren zu entfernen. Der Markraum wird mit Küretten und Kugelfräsen und anschließend mit der Jet-Lavage und Bürste gereinigt.

Die Jet-Lavage wird während der gesamten Operation immer wieder eingesetzt, durchaus unter Zusatz von Antiseptika wie z. B. Lavasept ${ }^{\circledR}$.

Auch pfannenseitig richtet sich die Schwierigkeit und Technik der Implantatentfernung nach deren Verankerungsmethode. Eine Auswahl unterschiedlicher Spezialmeißel und besonderer Explantationsinstrumente erleichtert die Arbeit und schont die Knochensubstanz. Die Säuberung des Implantatlagers im Azetabulum erfolgt analog zum Femur mit scharfen Instrumenten wie Kürette und Kugelfräse. Nach einer abschließenden Kontrolle auf Infektresiduen und dem ausgiebigen Gebrauch der Jet-Lavage beginnt die saubere Phase.

Erst jetzt startet die systemische Antibiotikatherapie, weil während des Débridements aus allen relevanten Regionen (Synovia, Azetabulum, Femureingang, Prothesenschaftspitze) Proben zur bakteriologischen Testung entnommen werden. So kann eine eventuelle Abweichung von der präoperativen Testung erkannt und die systemische Antibiose ggf. angepasst werden. Handschuhe, Bauchtücher und Saugerspitze werden gewechselt.

Ein kompletter Wechsel der Instrumente wäre ideal.

Jetzt erfolgt die Implantation einer geeigneten Prothese unter topischer Antibiose. Bei der Wahl der Prothese muss ein Kompromiss gefunden werden zwischen möglichst sicherer Verankerung und damit langer Prothesenstandzeit einerseits und Revisionsmöglichkeit ohne wesentliche Behinderung bei der Explantation im Falle einer Infektpersistenz andererseits.

Die Wunde wird über Drainagen primär verschlossen. Die Nachbehandlung ähnelt der bei aseptischen Revisionen. In problematischen Fällen ist die Rehabilitation vorsichtig und mit Rücksicht auf die Weichteilsituation ggf. deutlich langsamer anzustreben. Eine engmaschige Kontrolle des CRP ist obligat, wobei es im Laufe von 2-8 Wochen zu einer Normalisierung der Werte kommen sollte.
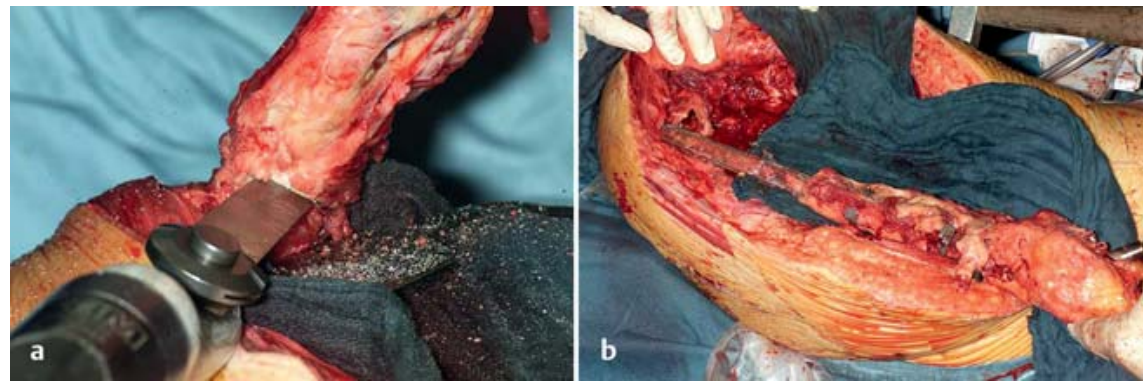

Abb. $\mathbf{6}$ a und b Radikale Explantation einer Sternprofilprothese bei erheblich geschädigtem proximalem Femur.

Beim zweizeitigen Vorgehen ist die gesamte 1. Phase identisch. Dann wird statt der Prothese ein Antibiotikumträger aus Zement implantiert. Der Wundverschluss erfolgt ebenfalls primär.

Nach einem individuellen Intervall, welches 4 Wochen zur Konsolidierung des Operationsgebiets nicht unterschreiten sollte, kann reimplantiert werden.

Meistens wird so lange gewartet, bis sich die CRP-Werte normalisiert haben. Eine Punktion in Anwesenheit eines Antibiotikaspacers und unter systemischer Therapie kann die Keimlage nicht sicher klären.

Ein neuerliches Débidement der Weichteile und der Knochenlager ist erforderlich, da trotz negativer Laborbefunde Keime persistieren können. Die zementierte Implantation der neuen Prothese bietet den Vorteil, eine gezielte topische Antibiose anzuwenden. Diese Möglichkeit wird bei Implantation einer zementfreien Prothese verschenkt. Zudem erfordert eine zementfreie Reimplantation in aller Regel eine weiter distal gelegene Verankerung. Im Revisionsfall wird so weitere Knochensubstanz geschädigt. Besonders distal verankerte Implantate mit sternförmigem Querschnitt lassen sich nicht ohne Femurfensterung oder Spaltung entfernen (Abb.6). Die dabei entstehenden Schäden haben erhebliche negative Folgen für die suffiziente Verankerung weiterer Prothesen. Bei einer Infektrezidivrate von etwa $15 \%$ im Falle von ein- wie zweizeitigem Vorgehen sollte diese Problematik unbedingt Berücksichtigung finden.

Besonders am Kniegelenk, aber auch an der Hüfte kann durch die Infektion bzw. die operative Technik ein Weichteilschaden entstehen, der den erforderlichen primären Wundverschluss ohne Weiteres unmöglich macht. Dieser muss dann mittels plastisch-chirurgischer
Maßnahmen, wie z.B. eines Gastrocnemiuslappens (Abb. 7), durchgeführt werden $[10,12]$.

\section{Implantate}

Nicht selten ist auch beim septischen Prothesenwechsel die Implantation von Primärimplantaten möglich.

Der Operateur muss aber immer darauf vorbereitet sein, auch größere knöcherne Verluststrecken ersetzen zu können.

Diese Substanzverluste können präoperativ bereits radiologisch dargestellt sein. Ihr Ausmaß kann sich aber durch die erforderliche Resektion von infizierten Arealen erheblich vergrößern. Da eine knöcherne Rekonstruktion mit homologem Knochen in der Infektsituation problematisch ist, kann diese nur mithilfe spezieller Revisionsimplantate (Abb. 8) gelingen.

Deshalb muss immer eine ausreichend große Anzahl von verschieden großen Sekundär- und Tertiärimplantaten vorgehalten werden $[26,29]$.

Am Kniegelenk ist im Infektfall durch die erforderliche Radikalität des Débridements die Integrität des Kapsel-BandApparats gefährdet. Das Débridement darf wegen besorgter Rücksicht auf die Stabilität der Kollateralbänder keinesfalls weniger umfassend durchgeführt werden. Dies würde die Infektsanierung gefährden. Deshalb sind gekoppelte Implantate, die abgesehen vom Streckapparat keine Restfunktion des Kapsel-BandApparats erfordern, unverzichtbar. Sie gewährleisten zuverlässig die nötige Stabilität. Je nach Erfordernis sind diese auch als Sekundärimplantate mit verlängerten femoralen und tibialen Schäften oder als Tertiärimplantat im Sinne einer totalen Femurprothese verfügbar [11]. 


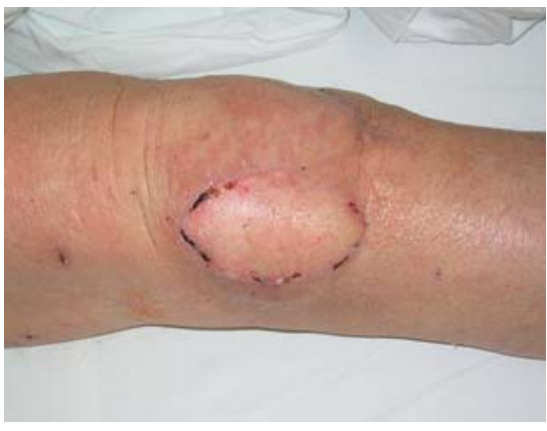

Abb.7 Gastrocnemiusplastik mit Vollhautinsel.

Bei Verlust des Streckapparats ist das Gelenk als Funktionseinheit verloren. Die Versteifung wird mit einem Arthrodesenagel durchgeführt, der intramedullär mit Zement verankert wird und deshalb eine topische Antibiotikatherapie ermöglicht.

Auch Längenverluste können so kompensiert werden und eine sofortige Vollbelastung ist möglich. Auch weitere Revisionen sind bei Verwendung des zementierten Nagels unproblematisch.

\section{Komplikationsmanagement}

Das Krankheitsbild und die chirurgische Therapie der periprothetischen Infektion sind komplikationsträchtig und risikoreich.

Die zwar seltene aber lebensbedrohliche generalisierte Keimaussaat im Sinne der Sepsis führt bei verzögerter Behandlung auch heute zum Tode. Der schlechte Allgemeinzustand des septischen Patienten darf nicht zur Verzögerung der Operation führen wegen vermeintlicher Inoperabilität, denn nur die sofortige chirurgische Intervention lässt eine Verbesserung zu.

Sie besteht mindestens aus der Eröffnung, Spülung und Drainage, wenn vertretbar, besser aus dem Débridement und der Prothesenentfernung. Bei meist unbekannter Keimlage wird eine zusätzliche antibiotische Therapie mit Breitenwirkung begonnen [15]. Diese wird entsprechend adaptiert, sobald die Keimlage durch die Exzidate der Revision aufgeklärt wird. Bei bekannter Keimlage ist durchaus auch bei Sepsis einzeitiges Vorgehen anzustreben.

Die Rate der Infektpersistenz nach dem 1. Wechsel liegt gemäß der Veröffentlichungen der Zentren bei ca. 15\% [1, 8, $22,27,36]$.
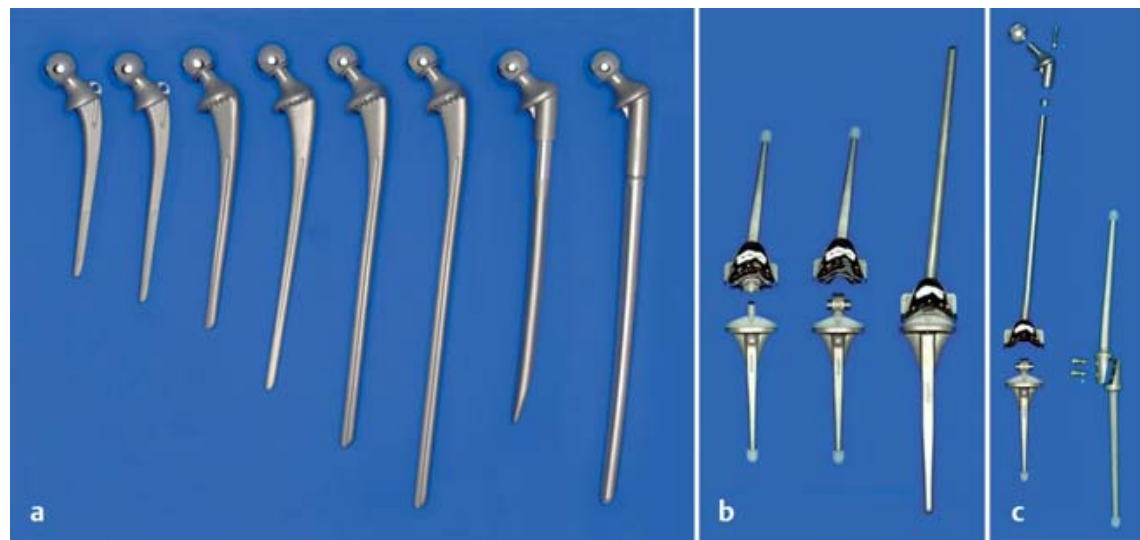

Abb. $\mathbf{8}$ a bis c Implantatpalette mit Revisionsimplantaten: a Hüfte, b Knie und c totale Femurprothese, Arthrodesenagel.

Im Allgemeinen ist sie vermutlich deutlich höher. Das therapeutische Vorgehen bei der Infektionspersistenz oder beim Rezidiv entspricht dem der primären Intervention. Verfahrenswechsel von einzu zweizeitig sind möglich, ohne dass hierdurch der Erfolg gesichert wäre.

Am Hüftgelenk ist die Luxationsrate nach septischem gegenüber aseptischem Wechsel stark erhöht.

Ursächlich dafür sind die hüftgelenksnahen Weichteilschädigungen, besonders der teilweise oder komplette Verlust der Abduktorenmuskulatur mit resultierender lateraler Instabilität. Antiluxationssicherungsringe können auf das Pfannenimplantat aufgeschraubt werden und diese Gefahr vermindern. Die äußerlichen Orthesen bieten in der Regel keine ausreichende Stabilität. Eine genaue Schulung der Patienten hinsichtlich zu vermeidender, luxationsgefährdender Bewegungen ist wichtig, in der Umsetzung jedoch schwierig. Dabei ist besonders darauf hinzuweisen, Manipulationen an den Füßen zu unterlassen und stattdessen Hilfe in Anspruch zu nehmen.

Das ebenfalls aus der Glutaealinsuffizienz resultierende Trendelenburg'sche Hinken sollte durch eine kontralateral getragene Gehhilfe ausgeglichen werden.

Bei allen Revisionsoperationen ist die anatomische Situation z.T. erheblich verändert und damit die Identifikation der verschiedenen Strukturen erschwert. Dies gilt besonders nach vielfachen Voroperationen und erst recht bei Infektionen. Der N. ischiadicus ist dadurch stark gefährdet und Verletzungen sind häufiger als im Regelfall.
Die möglichen Nebenwirkungen der systemisch verabreichten Antibiotika auf die inneren Organe sind zu bedenken, die Funktionswerte zu kontrollieren und die Dosierungen bei Funktionsstörungen von Leber und Niere anzupassen.

\section{Schlussfolgerung}

Die unstrittige Problematik in der Diagnostik und Therapie der periprothetischen Infektion erfordert bei bekanntem Konzept ein hohes Maß an Disziplin zum erfolgreichen Management dieser Komplikation. Primär sind eine zeitgemäße Prophylaxe durch Ausschaltung vermeidbarer Risikofaktoren, steriles, weichteilschonendes operatives Vorgehen sowie eine perioperative antibiotische Prophylaxe [15] entscheidend, um die Infektionsraten gering zu halten. Entsteht ein Infektverdacht, ist zügig und zielgerichtet vorzugehen. CRP-Bestimmung und Punktion des Gelenks sind sofort umzusetzen. Der Keimnachweis sichert die Diagnose und das Antibiogramm ist unverzichtbarer Bestandteil des Therapiekonzepts.

Die Umsetzung der bekannten Behandlungsprinzipien sollte in Zukunft erkennbar werden.

Weichteilrevisionen, Teilwechsel und Einlage von unwirksamen Antibiotikaträgern verschlechtern die Gesamtsituation. Der Behandlungserfolg und die Höhe der Behandlungskosten werden dadurch negativ beeinflusst. Die antibiotische „Anbehandlung“ oder „Abschirmung“ sind als Vorgehen strikt abzulehnen. Bei temporären Bakteriämien, wie sie in Verbindung mit Zahnbehandlungen auftreten können, muss jedoch bei Endoprothesenträgern eine antibiotische Prophylaxe, z.B. mit einem oralen Ampicillin, durchgeführt werden. 


\section{Literatur}

${ }^{1}$ Buechel FF, Femino FP, D'Alessio J. Primary exchange revision arthroplasty for infected total knee replacement: a long-term study. Am J Orthop 2004; 33: 190-198

2 Buchholz HW, Engelbrecht H. Über die Depotwirkung einiger Antibiotika bei Vermischung mit dem Kunstharz Palacos. Chirurg 1970; 41: 511-515

${ }^{3}$ Buchholz HW, Gartmann H-D. Infektionsprophylaxe und operative Behandlung der schleichenden tiefen Infektion bei der totalen Endoprothese. Chirurg 1972; 43: 446-453

${ }^{4}$ Buchholz HW, Elson RA, Engelbrecht $E$ et al. Management of deep infection of total hip replacement. J Bone Joint Surg [Br] 1981; 63 : 342-353

${ }^{5}$ Buttaro MA, Gimenez MI, Greco G et al. High active local levels of vancomycin without nephrotoxicity from impacted bone allografts in 20 revision hip arthroplasties. Acta Orthop 2005: 76: 336-340

${ }^{6}$ Costerton JW, Geesey GG, Cheng K-J. How bacteria stick. Sci Am 1978; 238: 86-95

7 Crockarell JR, Hanssen $A D$, Osmon $D R$ et al. Treatment of infection with debridement and retention of the components following hip arthroplasty. J Bone Joint Surg [Am] 1998; 80: 1306-1313

${ }^{8}$ Duncan CP, Masri BA. The role of antibioticloaded cement in the treatment of an infection after a hip replacement. J Bone Joint Surg [Am] 1994; 76: 1742-1751

9 Durbhakula SM, Czajka J, Fuchs MD et al. Antibiotic-loaded articulating cement spacer in the 2-stage exchange of infected total knee arthroplasty. J Arthroplasty 2004; 19: 768 774

${ }^{10}$ Fansa H, Plogmeier K, Schenk K et al. Die Deckung ausgedehnter Weichteildefekte be infizierten Knieendoprothesen durch Gastrocnemiuslappen. Chirurg 1998; 69: 1238 1243

${ }^{11}$ Friesecke C, Plutat J, Block A. Revision arthroplasty with use of a total femur prosthesis J Bone Joint Surg [Am] 2005; 87: 2693-2701

12 Friesecke C, Wodtke J. Management des Protheseninfektes. Chirurg 2008; 79: 777-794

${ }^{13}$ Friesecke C, Wodtke J. Die periprothetische Kniegelenksinfektion. Orthopäde 2006; 35 : 937-945

14 Frommelt L. Prinzipien der Antibiotikabehandlung bei periprothetischen Infektionen. Orthopäde 2004; 33: 822-826
${ }^{15}$ Frommelt L. Systemische Antibiotikatherapie bei Infektionen im Skelettsystem. In: Hendrich C, Frommelt L, Eulert J, Hrsg. Septische Knochen- und Gelenkchirurgie. Berlin, Heidelberg, New York: Springer; 2004: 98-105

${ }^{16}$ Fuerst M, Fink B, Ruther W. The value of preoperative knee aspiration and arthroscopic biopsy in revision total knee arthroplasty. Z Orthop Ihre Grenzgeb 2005; 143: 36-41

17 Geipel U, Hermann M. Das infizierte Implantat. Teil 1. Bakteriologie. Orthopäde 2004; 33: $1411-1428$

18 Gristina AG. Biomaterial-centered infection: microbial adhesion versus tissue integration. Sci Am 1987; 237: 1588-1595

${ }^{19}$ Hoad-Reddick DA, Evans CR, Norman P et al. Is there a role for extended antibiotic therapy in a two-stage revision of the infected knee arthroplasty. J Bone Joint Surg [Br] 1987; 87: 171-174

20 Hofmann AA, Goldberg T, Tanner AM et al. Treatment of infected total knee arthroplasty using an articulating spacer: 2 - to 12 -year experience. Clin Orthop Relat Res 2005; 430: $125-131$

${ }^{21}$ Kraemer WJ, Saplys R, Waddell JP et al. Bone scan, gallium scan, and hip aspiration in the diagnosis of infected total hip arthroplasty. J Arthroplasty 1993; 8: 611-616

22 Langlais F, Lambotte JC, Thomazeau H. Treatment of infected total hip replacement. In: Lemaire R, Herom F, Scott J, Villar R, eds. European instructional course lecture 6. London: The British Society of Bone and Joint Surgery; 2003: 158-167

23 LaPorte DM, Waldman BJ, Mont MA et al. Infections associated with dental procedures in total hip arthroplasty. J Bone Joint Surg [Br] 1999; 81: 56-59

24 Lautenschläger EP, Marshall GW, Marks KE et al. Mechanical strength of acrylic bone cements impregnated with antibiotics. J Biomed Mater Res 1976; 10: 837-845

25 Lodenkämper $H$, Lödenkämper U, Trompa $K$. Über die Ausscheidung von Antibiotika aus dem Knochenzement Palacos (eigene Erfahrungen aus bakteriologischer Sicht nach 10jähriger Anwendung in der Gelenkersatzchirurgie). Z Orthop 1982; 120: 801-805

${ }^{26}$ Nieder E, Friesecke C, Plutat J. Ergebnisse von 73 Implantationen der Sattelprothese Mk II Endo-Modell in der Revisionsalloarthroplastik. In: Kerschbaumer F, Rehart S, Nieder E, Hrsg. Die Hüftendoprothese in komplizierten Fällen. Stuttgart, New York: Thieme; 1995: 180-195

27 Sanchez-Sotelo J, Berry DJ, Hanssen AD et al. Midterm to long-term follow up of staged reimplantation for infected hip arthroplasty. Clin Orthop Relat Res 2009; 467: 219-224
${ }^{28}$ Scher DM, Pak K, Lonner JH et al. The predictive value of indium-111 leucocyte scans in the diagnosis of infected total hip, knee, or resection arthroplasties. J Arthroplasty 2000; 15: 295-300

29 Siegel A, Rückert U, Wenzl M. Seltene Indikation zur Anwendung einer einfachen Hüftgelenkendoprothese mit übergroßem Kopf nach zahlreichen unbefriedigenden Wechseloperationen. Chirurg 1999; 70: 1353-1356

${ }^{30}$ Steinbrink K, Mella-Schmidt C. Stellenwert der Spül-Saugdrainage bei der Behandlung des Frühinfekts von Gelenkimplantaten. Chirurg 1989; 60: 791-794

31 Steinbrink K, Frommelt L. Behandlung der periprothetischen Infektion der Hüfte durch einzeitige Austauschoperation. Orthopäde 1995; 24: 335-343

32 Taylor T, Beggs I. Fine needle aspiration in infected hip replacements. Clin Radiol 1995; 50: 149-152

33 Wahlig H, Dingeldein E, Buchholz HW et al. Pharmacokinetic study of gentamycin-loaded cement in total hip replacement. J Bone Joint Surg [Br] 1984; 66: 175-179

${ }^{34}$ Winkler H, Stoiber A, Kaudela $K$ et al. One stage uncemented revision of infected total hip replacement using cancellous allograft bone impregnated with antibiotics. J Bone Joint Surg [Br] 2008; 90: 1580-1584

35 Wodtke J, Löhr JF. Das infizierte Implantat. Orthopäde 2008; 37: 257-269

${ }^{36}$ Wodtke J, Jonen V, Briem D. Analysis and results of 282 one-stage exchanges for periprosthetic infection of the hip and knee. Vienna: EBJIS 28th annual meeting; September 2009

37 Zimmerli W, Trampuz A, Ochsner PE. Periprosthetic-joint infections. N Engl J Med 2004; 351: 39-48

38 Zimmerli W, Lew PD, Waldvogel FA. Pathogenesis of foreign body infection. Evidence for a local granulocyte defect. J Clin Invest 1984; 73: 1191-200

\section{Dr. med. Christian Friesecke}

LOA

Dr. med. Joachim Wodtke

LOA

ENDO-Klinik Hamburg GmbH

Holstenstraße 2

22767 Hamburg

christian.friesecke@endo.de 